\title{
Turbulent swirling flow in a model of a uniflow-scavenged two-stroke engine
}

\section{Journal Article}

Author(s):

Ingvorsen, Kristian M.; Meyer, Knud Erik; Walther, Jens Honoré (i); Mayer, Stefan

Publication date:

2013-03

Permanent link:

https://doi.org/10.3929/ethz-b-000066114

Rights / license:

In Copyright - Non-Commercial Use Permitted

Originally published in:

Experiments in Fluids 54(3), https://doi.org/10.1007/s00348-013-1494-6 


\title{
Turbulent swirling flow in a model of a uniflow-scavenged two-stroke engine
}

\author{
K. M. Ingvorsen · K. E. Meyer · J. H. Walther • \\ S. Mayer
}

Received: 14 December 2012/Revised: 22 February 2013/ Accepted: 26 February 2013/Published online: 14 March 2013

(C) Springer-Verlag Berlin Heidelberg 2013

\begin{abstract}
The turbulent and swirling flow of a uniflowscavenged two-stroke engine cylinder is investigated using a scale model with a static geometry and a transparent cylinder. The swirl is generated by 30 equally spaced ports with angles of $0^{\circ}, 10^{\circ}, 20^{\circ}$, and $30^{\circ}$. A detailed characterization of the flow field is performed using stereoscopic particle image velocimetry. Mean fields are calculated using both a fixed coordinate system and a coordinate system based on the instantaneous flow topology. Timeresolved measurements of axial velocity are performed with laser Doppler anemometry, and power spectra are calculated in order to determine vortex core precession frequencies. The results show a very different flow dynamics for cases with weak and strong swirl. In the strongly swirling cases, a vortex breakdown is observed. Downstream of the breakdown, the vortex becomes highly
\end{abstract}

Electronic supplementary material The online version of this article (doi:10.1007/s00348-013-1494-6) contains supplementary material, which is available to authorized users.

K. M. Ingvorsen · K. E. Meyer · J. H. Walther $(\bowtie)$

Department of Mechanical Engineering, Technical University of Denmark, Kgs. Lyngby, Denmark

e-mail: walther@mavt.ethz.ch; jhw@mek.dtu.dk

K. M. Ingvorsen

e-mail: kmin@mek.dtu.dk

J. H. Walther

Computational Science and Engineering Laboratory,

ETH Zürich, Zürich, Switzerland

S. Mayer

MAN Diesel \& Turbo SE, Copenhagen, Denmark

e-mail: Stefan.Mayer@man.eu concentrated and the vortex core precesses around the exhaust valve, resulting in an axial suction effect at the vortex center. Mean fields based on the instantaneous flow topology are shown to be more representative than mean fields based on a fixed coordinate system in cases with significant variations in the swirl center location.

\section{Introduction}

Large two-stroke uniflow marine diesel engines are the de facto standard technology for the propulsion of large commercial vessels. A key process for this type of engine is the scavenging process (Schweitzer 1949). In a uniflowscavenged engine, scavenge ports located in the lower part of the cylinder liner are uncovered by the piston as it moves toward the bottom dead center. Compressed air enters through the ports filling the cylinder for the next engine cycle. The introduction of fresh air forces the exhaust gases out through one or more exhaust valves located in the cylinder head. The scavenge ports are cut at an angle with respect to the radial direction to create a swirling flow. The purpose of the swirl is to stabilize the scavenging process, control the heat transfer to and from the cylinder surfaces (piston head, liner, cylinder head, and valve), and later to enhance the mixing of air and injected fuel. The uniflow scavenging process is thus characterized by a transient (opening/closing of ports) confined port-generated turbulent swirl flow. A suboptimal scavenging design can lead to a poor scavenging efficiency, high fuel consumption, increased emissions, improper cooling of cylinder surfaces, and large cycle-to-cycle variations.

Due to the large costs and difficulties related to tests on full-scale engines, accurate computational fluid dynamics (CFD) models are needed in order to optimize the in- 
cylinder processes. However, due to the complex physical phenomena, especially related to the turbulent swirling flow (Craft et al. 2008), experimental data are needed in order to evaluate the accuracy of the numerical predictions. The present work is therefore concerned with an experimental investigation of the scavenging flow using a scale model of a uniflow-scavenged cylinder. The main goals are to obtain accurate and high-resolution data suitable for CFD validation purposes and furthermore to obtain insight into the physics of the in-cylinder flow that can aid the engineer when setting up a CFD model.

Several earlier works have been devoted to experimental investigations of the uniflow scavenging process. Measurements in static models have been performed using a pitot device by Percival (1955), laser Doppler anemometry (LDA) by Sung and Patterson (1982) and Sher et al. (1991), and particle image velocimetry (PIV) by Haider et al. (2012). Investigations in dynamic models have been performed by, for example, Ohigashi et al. (1960) and Nishimoto and Kamimoto (1984) measuring the shape front of scavenging air; Sanborn and Dedeoglu (1988), Dedeoglu (1990), and Litke (1999) with the use of singlecycle liquid models, and LDA measurements in a motored engine with compression by Nakagawa et al. (1990). Using a single cylinder test engine with a variable angle swirler, Wakuri et al. (1981) carried out fired engine tests to investigate the effect of swirl on fuel consumption and soot production.

In general, it is found that the introduction of swirl creates a velocity deficit near the cylinder center in the radial profiles of axial velocity (Percival 1955). The deficit is unwanted as it can result in a poorly scavenged central region (Dedeoglu 1990; Nakagawa et al. 1990). As the swirl strength is increased, the deficit gets wider and the centerline velocity decreases. If the degree of swirl exceeds a certain value, the axial centerline velocity obtains negative values, showing that a central toroidal recirculation zone (CTRZ) (Gupta et al. 1984) has been formed. The formation of a CTRZ in swirling flows is by many researchers perceived as the manifestation of a vortex breakdown. The vortex breakdown is a characteristic of strongly swirling flows and known from related studies such as the flow in swirl chambers (Sarpkaya 1971; Escudier et al. 1982), swirl-stabilized flames, and swirl combustors (Gupta et al. 1984). The shape of the tangential velocity profiles is less affected by the swirl strength. In general, the profiles are characterized by a region close to the center showing nearly solid-body rotation. This region ends approximately at the radial position corresponding to maximum tangential velocity. At larger radial positions, the tangential velocity is often observed to be constant, but secondary peaks in the profiles have also been reported, that is, the tangential velocity does not decay toward the wall.
For the present investigations, a scale model of a uniflow-scavenged cylinder is constructed. The model has a simplified geometry compared to real engines with a flat piston and a static exhaust valve and is axisymmetric (except for the 30 scavenge ports), thus simplifying the process of geometry and mesh generation for CFD simulations. The dimensions of the model are based on the T50MX test engine at MAN Diesel \& Turbo SE, Copenhagen, Denmark. The model has a transparent cylinder allowing for the use of non-intrusive optical measurement techniques. This is an important feature as swirling flows are known to be sensitive to disturbances caused by the introduction of, for example, pitot tubes and hotwire probes (Holman and Moore 1961). The model is fitted with a moving piston allowing for dynamic model tests. However, for the present work, the piston is fixed at the bottom dead center so that the swirling incylinder flow can be studied without the added complexity of the opening and closing of ports. This will make it possible to distinguish between phenomena related to the opening and closing of the ports and the inherent flow characteristics of the confined turbulent swirl flow in future dynamic investigations. Preliminary results from dynamic tests are reported by Ingvorsen et al. (2012). To investigate the effect of swirl strength, different port angles are tested: $0^{\circ}, 10^{\circ}, 20^{\circ}$, and $30^{\circ}$, corresponding to non-swirling, weak, medium, and strong swirl, respectively. In real engines, including the T50MX test engine, port angles around $20^{\circ}$ are used. The flow is investigated using both stereoscopic PIV (Raffel et al. 2007) and LDA (Albrecht et al. 2003). The PIV technique allows for measurements of the instantaneous three-component velocity fields across the cylinder cross section at a low sampling rate, whereas the LDA produces timeresolved point measurements. Hence, the combination of the two techniques provides a good basis for analyzing the in-cylinder flow.

\section{Methodology}

\subsection{Experimental setup}

A scale model of a simplified uniflow-scavenged cylinder is constructed for the present study, see Fig. 1. The cylinder has an inner diameter of $D=190 \mathrm{~mm}$ and is made of glass of good optical quality. The piston has a flat surface and is positioned such that the piston surface is flush with the bottom of the ports. This piston position is chosen as it gives the most generic geometry and at the same time corresponds roughly to the geometry of an engine cylinder with the piston at bottom dead center. The cylinder is $5.00 \mathrm{D}$ long measured from piston surface to cylinder head. 


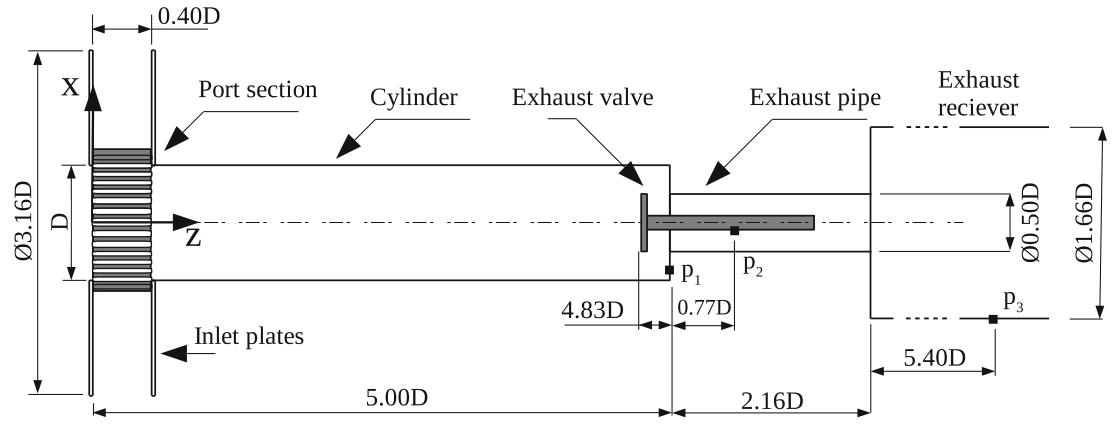

(a) Experimental model

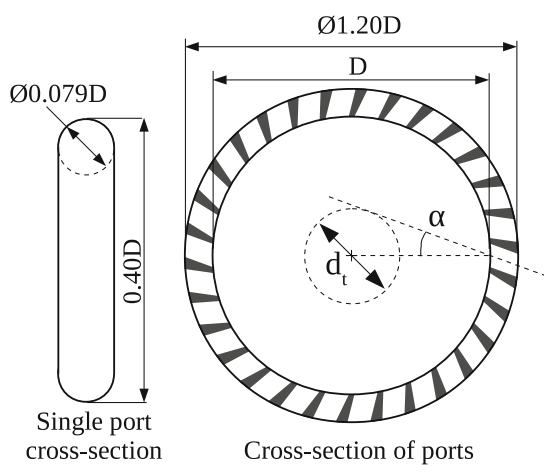

(b) Port details

Fig. 1 Sketches of the experimental model

The port section consists of $n_{p}=30$ equally spaced ports and has an outer diameter of $1.20 D$. Each port is a $0.079 D(15 \mathrm{~mm})$ wide slit milled into the cylinder wall at an angle $\alpha$ (see Fig. 1b). The port height is $0.40 D$, and the top and bottom of the ports are round. Four port sections with port angles of $\alpha=\left\{0^{\circ}, 10^{\circ}, 20^{\circ}, 30^{\circ}\right\}$ are used. The port angle can also be expressed as the ratio of the tangent circle diameter $d_{t}$ to cylinder diameter $D$. The ratio of diameters is related to the port angle by $d_{t} / D=\sin \alpha$ (c.f. Fig. 1b). Ambient air is guided to the ports by two $0.05 D$-thick parallel disks with a separation equal to the port height and a diameter of $3.16 \mathrm{D}$. The flow exits the cylinder through an exhaust pipe with a diameter of $0.50 D$. A simplified exhaust valve is placed in front of the exhaust pipe $0.17 D$ upstream the cylinder head. The valve consists of a disk (thickness $0.02 D$, diameter $0.50 D$ ) mounted on a circular rod (diameter $0.126 D$, length $1.34 D$ ). The valve opening, that is, the distance between the disk and the cylinder head, is $0.15 D$. The exhaust pipe has a length of $2.16 D$ and ends in a large cylindrical receiver (diameter $1.66 \mathrm{D}$, length $6.74 \mathrm{D}$ ). The receiver is connected to a frequency-controlled centrifugal fan, driving the flow through the model. In order to measure the flow rate through the model, an orifice flow meter is mounted downstream the fan. Static gauge pressure is measured in the model using three wall pressure tabs $\left(P_{i}, i=1,2,3\right)$. The first $\left(P_{1}\right)$ is located on the cylinder head at a radius of $r / D=0.41$, the second $\left(P_{2}\right)$ is on the valve rod $0.77 D$ downstream the inlet of the exhaust pipe, and the third $\left(P_{3}\right)$ is on the receiver wall $5.40 \mathrm{D}$ downstream the receiver inlet. The model is placed in a large tent, and experiments are performed using air at room temperature and atmospheric pressure. A Cartesian coordinate system is defined with origin at the center of the piston surface, the $z$ axis coinciding with the (horizontal) cylinder axis, and the $x$ axis in the perpendicular, horizontal direction.

\subsection{Flow parameters}

The flow in the model is characterized by the Reynolds number $R e$ and the swirl number $S_{w}$. The Reynolds number is given by

$R e=\frac{D W_{b} \mu}{\rho}$

where $\mu$ is the dynamic viscosity, $\rho$ is the density, and $W_{b}$ is the axial bulk velocity in the cylinder. Unless otherwise specified, measurements are carried out at a Reynolds number of $R e=50,000$. The large Reynolds number indicates that inertial forces dominate over viscous forces and that the in-cylinder flow is fully turbulent except very close to the walls. For each measurement series, the viscosity and density are calculated based on the measured temperature and barometric pressure. Using these values the flow rate is adjusted such that the target Reynolds number is achieved. For the reference temperature and pressure $\left(20^{\circ} \mathrm{C}, 1 \mathrm{~atm}\right)$, a Reynolds number of $R e=50,000$ corresponds to a bulk velocity of $W_{b}=3.96 \mathrm{~m} / \mathrm{s}$.

The swirl number $S_{w}$ quantifies the degree of swirl in a flow. Several definitions exist but the most common (Gupta et al. 1984) is given by

$S_{w}=\frac{2 F_{\theta, z}}{F_{z, z} D}$

where $F_{\theta, z}$ is the axial flux of angular momentum and $F_{z, z}$ is the axial flux of axial momentum. Accurate calculation of the swirl number given by Eq. (2) is in general impossible as the velocity and pressure fields are not known beforehand. A design swirl number $\Omega$ is therefore often introduced based on the flow geometry (Alekseenko et al. 1999). For the present geometry, $F_{\theta, z}$ is estimated by assuming that it is equal to the radial flux of angular momentum through the ports. 
$F_{\theta, z} \approx F_{\theta, r}=\rho \frac{D}{2} V_{\theta} \frac{n_{p} A_{p}}{\cos \alpha} V_{r}$

where $A_{p}$ is the cross-sectional area of a single port and $V_{r}$ and $V_{\theta}$ are the radial and tangential velocity in the ports. Assuming a uniform flow parallel to the ports, $V_{r}$ and $V_{\theta}$ are simply given by $V_{r}=\frac{A_{c y l}}{n_{p} A_{p}} W_{b} \cos \alpha$ and $V_{\theta}=\frac{A_{c y l}}{n_{p} A_{p}} W_{b} \sin \alpha$, where $A_{\text {cyl }}$ is the cross-sectional area of the cylinder. The axial flux of axial momentum is estimated by neglecting the pressure term and assuming a plug flow in the cylinder

$F_{z, z}=\rho W_{b}^{2} A_{\mathrm{cyl}}$

Applying continuity, the design swirl number can be expressed as

$\Omega=\frac{A_{\mathrm{cyl}}}{n_{p} A_{p}} \sin \alpha$

From Eq. (5) it is noted that the design swirl number can be changed both by varying the port angle and by changing the port exit area, for example, by partly blocking the ports with the piston. The design swirl number and ratio of tangent circle diameter to cylinder diameter are presented for the different port angles in Table 1 . The above analysis is in good agreement with the findings by Nakagawa et al. (1990) and Wakuri et al. (1981) showing that both the maximum tangential velocity and the angular momentum are nearly proportional to $\sin \alpha$.

\subsection{Pressure measurements}

The pressure difference across the orifice plate and the gauge pressure at the pressure tabs are measured with a Furness controls FCO510 digital micromanometer. The total uncertainty of the bulk velocity measurement based on the orifice meter is estimated to be $\pm 0.5 \%$. The timeaveraged gauge pressure at the pressure tabs is denoted $P_{i}$, where $i=\{1,2,3\}$ refers to the pressure tab positions (Fig. 1a). The total uncertainty of the gauge pressures is estimated to be $\pm 2 \%$ of the pressure reading.

\subsection{A note on CFD simulations}

Concerning the setup of CFD simulations of the flow in the present model, the authors have the following recommendations on boundary conditions. Pressure boundaries should be used for both inlet and outlet. The inlet boundary

Table 1 The design swirl number $\Omega$ and ratio of tangent circle diameter to cylinder diameter $d_{t} / D$ for the different port angles $\alpha$

\begin{tabular}{lllll}
\hline$\alpha$ & $0^{\circ}$ & $10^{\circ}$ & $20^{\circ}$ & $30^{\circ}$ \\
$d_{t} / D$ & 0.00 & 0.17 & 0.34 & 0.50 \\
$\Omega$ & 0.00 & 0.15 & 0.30 & 0.43 \\
\hline
\end{tabular}

should be located in the ambient air and cover the entrance to the inlet plates, for example, by using a torus shape or similar. The flow at this location can be assumed laminar, and the pressure constant and equal to the ambient pressure. The outlet boundary should be a cross section of the exhaust receiver at the axial position corresponding to the location of the $P_{3}$-pressure tab, c.f. Fig. 1a. Preliminary LES simulations indicate that the pressure at this axial position is close to constant over the receiver cross section. The measured static wall pressure, given in Sect. 3.1, can thus be used as boundary value.

\subsection{Laser Doppler anemometry}

The laser Doppler anemometry (LDA) system used consists of a Dantec two-component FiberFlow optics mounted on a three-axis traverse, two Dantec BSA enhanced processors, and Dantec BSA Flow Software. The laser is a $4 \mathrm{~W}$ Coherent Innova 90 Argon-Ion laser. Only the $514.5 \mathrm{~nm}$ wavelength is used, measuring the axial velocity component. The system is operating in backscatter mode using a front lens of $600 \mathrm{~mm}$, resulting in a probe volume of $2.5 \mathrm{~mm} \times 0.25 \mathrm{~mm} \times 0.25 \mathrm{~mm}$ with the long side parallel to the $x$ axis. Due to optical distortions created by the curved glass pipe, measurement positions are limited to a horizontal plane going through the cylinder axis $(y=0)$. The ambient air near the inlet plates is seeded with Diethyl-hexyl-sebacate (DEHS) particles generated with a Laskin nozzle. The generated particles have diameters in the range $0.1-2.0 \mu \mathrm{m}$ which corresponds to a maximal particle relaxation time of $\tau_{s}=d_{p}^{2} \frac{\rho_{p}}{18 \mu} \approx 10 \mu \mathrm{s}$, where $\rho_{p}$ is the particle density and $d_{p}$ is the particle diameter (Raffel et al. 2007). Analysis of the power spectra acquired with LDA, cf. Sect. 3.6, shows that the majority of the energy corresponds to timescales of $1 \mathrm{~ms}$ or higher. The particles are thus expected to follow the flow faithfully.

\subsection{Stereoscopic particle image velocimetry}

Stereoscopic particle image velocimetry (PIV) measurements are carried out using a commercial PIV system. The two cameras are 12-bit gray scale cameras (Dantec Dynamics HiSense Mk II) with a chip size of $1,344 \mathrm{px} \times$ $1,024 \mathrm{px}$ and a pixel pitch of $6.45 \mu \mathrm{m}$. The cameras are fitted with $60-\mathrm{mm}$ lenses using Scheimpflug mounts. To illuminate the flow field, a pulsed double cavity Nd:YAG laser fitted with light sheet optics is used. The light sheet thickness is 2-3 $\mathrm{mm}$ and the maximum laser power is used, corresponding to approximately $200 \mathrm{~mJ}$ per pulse. The seeding particles are the same as in the LDA measurements.

One camera is mounted on the same side as the laser, and the second camera is mounted on the opposite side of 
Table 2 Axial position of the PIV measurement planes

\begin{tabular}{ll}
\hline Pos. & $z / D$ \\
\hline 1 & 0.58 \\
2 & 1.00 \\
3 & 1.50 \\
4 & 2.00 \\
5 & 2.50 \\
6 & 3.00 \\
7 & 3.50 \\
8 & 4.00 \\
9 & 4.50 \\
10 & 4.80 \\
\hline
\end{tabular}

the cylinder. The camera on the same side as the laser receives significantly less light than the second camera due to its backward scatter position. To account for this, the $F$-number is set to 8 for the camera in backward scatter position and 11 for the camera in forward scatter position. PIV measurements are carried out for ten cross-sectional planes. The axial locations of the measurement planes are given in Table 2. The cameras are mounted on the downstream side of the light sheet for measurements at the interval $z / D=[0.58,4.00]$, and on the upstream side for measurements close to the valve $z / D=\{4.50,4.80\}$. The cameras and the laser are mounted on a traversing system aligned with the cylinder axis. This allows measurements to be performed at several axial positions using the same camera calibration.

The camera calibration and processing of particle images are carried out using Dantec DynamicStudio ver. 3.3. Calibration images are obtained using a 2D calibration target positioned in the cylinder cross section. Thirteen image pairs corresponding to a \pm 5 -mm interval around the target are recorded by moving the cameras in small axial increments. The calibration is refined by carrying out a disparity correction to correct for small misalignments between the calibration target and the light sheet. The particle images are processed using a three-step adaptive correlation algorithm with final interrogation windows of $32 \times 32$ pixels and $50 \%$ overlap, resulting in a data spacing of $0.01 D$. Spurious vectors are removed using a validation scheme based on the local median and replaced with interpolated data. For positions close to the scavenge ports and exhaust valve, the model geometry appears in the background of the particle images. To enhance the quality of the particle images, background images are subtracted.

\subsubsection{Post-processing}

For each axial position 1,000 instantaneous velocity fields $v_{i}(x, y)$ are recorded with a sampling rate of $6 \mathrm{~Hz}$, where $v_{i}, \mathrm{i}=1,2,3$ corresponds to velocity components in the $x, y$, and $z$ direction $(u, v, w)$, respectively. The timeaveraged velocity fields $V_{i}(x, y)$ and rms-velocity fields $V_{i, \mathrm{rms}}(x, y)$ are then calculated as the ensemble average and standard deviation. An example of the time-averaged velocity fields is shown in Fig. 2. The field of view covers an almost rectangular region where the corners are close to the cylinder wall. The time-averaged and rms-velocity fields are close to axisymmetric with the axis of symmetry near the cylinder axis. It is therefore convenient to describe the mean flow using a cylindrical coordinate system with coordinates $r, \theta, z$. In the $0^{\circ}$ port angle case, the origin of the cylindrical coordinate system is coincident with the Cartesian. For the swirling flow cases the origin is moved such that it coincides with the mean swirl center at the given $z$-position. Similar procedures have been used by other authors (Mattner et al. 2002; Haider et al. 2012). The mean swirl center is found to be within a few percent of $D$ from the cylinder axis for all measured positions. Radial profiles of the time-averaged velocities $V_{r}, V_{\theta}, V_{z}$ are obtained by averaging in the $\theta$ direction. This is in practice achieved by performing a radial binning of the velocities. Figure 3 shows the time-averaged velocities as a function of radial position (gray dots), together with the profiles obtained by binning (black curves). It is noted that mean velocity field is well described by the radial profiles as only a small amount of scatter is observed.

Inspection of instantaneous velocity fields from the swirling cases shows that the swirl center at a given axial position is not stationary but moves around a mean position. The unsteady nature of the swirl center location is a well-known phenomenon for swirling flows. The movement can be both random in nature (e.g., Escudier et al. 2006; Huang and Tsai 2001) and the effect of a precessing vortex core (Gupta et al. 1984). Using a fixed coordinate system in cases with significant swirl center motion will

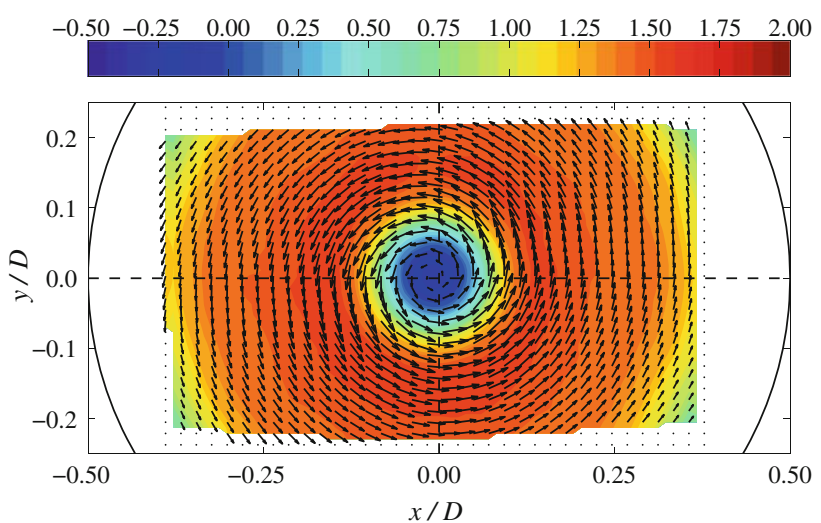

Fig. 2 Time-averaged velocity fields at $z / D=1.0$ for $\alpha=20^{\circ}$. Inplane velocities $(U, V)$ are shown by vectors, and normalized axial velocity $W / W_{b}$ is shown by the contour plot. Every second vector is shown in each direction, and $W_{b}$ corresponds to a vector length of $0.025 D$ on the axis 


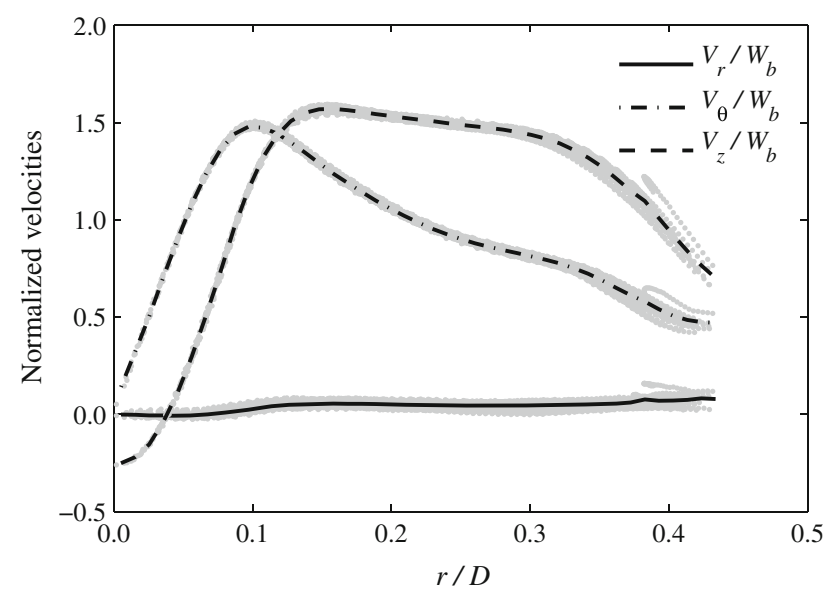

Fig. 3 Comparison of time-averaged velocities (gray dots) and radial profiles obtained by binning (black curves). Data from $z / D=1.00$ for the $\alpha=20^{\circ}$ case

result in a smearing effect on the mean fields and high values in the rms-fields. To investigate this effect, additional mean fields are calculated based on the instantaneous swirl centers. To calculate these mean fields, the instantaneous location of the swirl center is determined for all $N=1,000$ snapshots at a given $z$-position. The mean field is then obtained by averaging the velocity fields expressed using a coordinate system moved to the instantaneous swirl centers. Radial profiles are then created by radial binning as described above. To avoid problems near the walls caused by the difference between the swirl center and the geometric center, all profiles are cut at $r>0.45 D$.

To determine the position of the swirl center $\left(x_{\mathrm{sw}}, y_{\mathrm{sw}}\right)$, the function $C$ is introduced as

$C(x, y)=\frac{\omega_{z}(x, y)}{V_{u, v}(x, y)+0.05 W_{b}}$

where $\omega_{z}=-\frac{\partial u}{\partial y}+\frac{\partial v}{\partial x}$ is the axial component of vorticity, $V_{u, v}=\sqrt{u^{2}+v^{2}}$ is the in-plane velocity magnitude, and the term $0.05 W_{b}$ is included in order to avoid division by zero. $C$ will in general have its maximum value at the swirl center, due to the fact that the axial vorticity will be large and the in-plane velocity magnitude will be small at the swirl center. The swirl center position is determined by finding the area-averaged center of gravity for data points corresponding to $C / C_{\max }>0.95$. When determining the swirl centers of the instantaneous velocity fields, the velocity fields are smoothed using a moving-average filter with a window size of $0.05 D \times 0.05 D$ before $C$ is evaluated.

\subsubsection{Validity of profiles}

The accuracy of the obtained velocity profiles is investigated both by testing repeatability and by comparing with reference LDA measurements. To test for repeatability, a second PIV measurement series is carried out. The new measurements are carried out after having disassembled and reassembled major parts of the flow rig and optical setup and are based on a new camera calibration. The radial profiles of $V_{r}, V_{\theta}$, and $V_{z}$ are measured at six axial positions. Comparison of the profiles shows a high repeatability with a mean discrepancy of $1 \%$ of $W_{b}$ and $95 \%$ of the discrepancies less than $5 \%$ of $W_{b}$.

As mentioned in Sect. 2.5, the LDA is limited to measuring the axial velocity component in the horizontal plane through the cylinder center. To create radial profiles, the probe volume is therefore traversed in the $x$-direction, the obtained profile shifted such that it becomes symmetric, and measurements for positive and negative $x$-positions are averaged. Figure 4 shows the comparison of the LDA profiles and PIV profiles at two axial positions. Good agreement is observed both for the repeated PIV profiles and for the LDA profiles with a mean discrepancy between LDA and PIV measurements of $3 \%$ of $W_{b}$ and $95 \%$ of the discrepancies less than $9 \%$ of $W_{b}$. The slightly larger discrepancies observed between LDA and PIV compared to the repeated PIV measurements are attributed to the fact that the LDA profiles are measured along the geometric axis which in general does not go exactly through the mean swirl center.

The effect of Reynolds number on the non-dimensional profiles is also investigated using LDA measurements. Profiles of the axial velocity at $z / D=1.0$ are measured for Reynolds numbers of 35,000, 50,000, and 80,000 (not shown). It is found that the non-dimensional mean velocity profiles are independent of all three Reynolds numbers, whereas the non-dimensional rms-profiles are independent of Reynolds numbers for $R e=50,000$ and 80,000, while the profiles for $R e=35,000$ show slightly higher values.

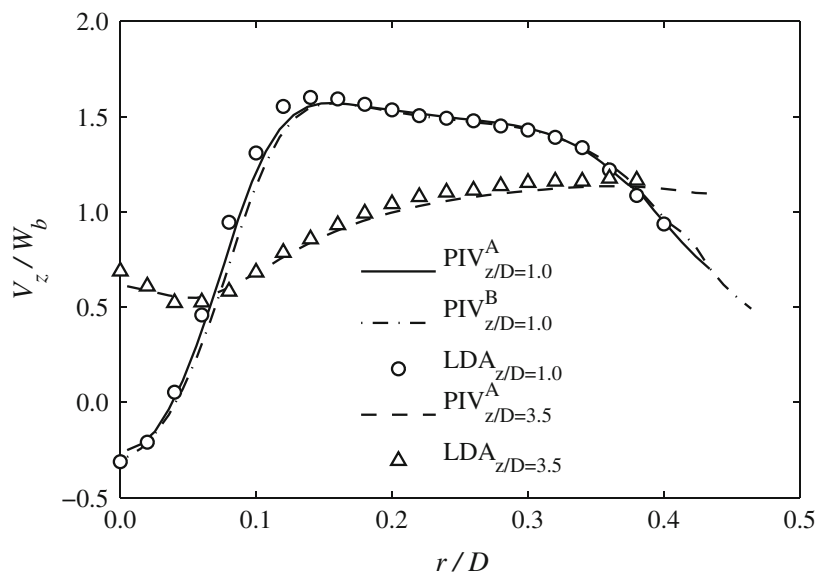

Fig. 4 Comparison of axial velocities measured using PIV and LDA for a port angle of $\alpha=20^{\circ}$. Superscripted 'A' and 'B' refer to the repeated measurement series 


\section{Results}

\subsection{Pressure measurements}

The time-averaged static gauge pressures at the positions shown in Fig. 1a are presented in Table 3. To interpret the pressure measurements, the following is noted. The pressure difference across the ports is the main contribution to $P_{1}$, the pressure difference created by the valve disk can be expressed as $P_{2}-P_{1}$, the pressure difference created by the sudden expansion from exhaust pipe to exhaust receiver can be expressed as $P_{3}-P_{2}$, and the total pressure difference across the model is given by $P_{3}$.

It is observed that the pressure difference across the ports increases a function of the port angle. This is expected and in agreement with the findings by Percival (1955). The pressure difference over the valve is approximately 16 times higher than the pressure difference over the ports, and the lowest pressure differences are observed for the $10^{\circ}$ and $20^{\circ}$ cases. A pressure recovery is observed for the transition from exhaust pipe to receiver. The recovery shows a linear dependence of port angle, resulting in the largest recovery for the $30^{\circ}$ case. This behavior is expected to be the result of the swirl stabilizing the flow and forcing it outward in the exhaust receiver, thus increasing the diffuser effect. In general, it can be concluded that the pressure difference over the ports is small compared to pressure differences related to the exhaust geometry; this agrees with observations by Wakuri et al. (1981). $P_{3}$ shows that swirling flows result in a smaller pressure difference from the model inlet to exhaust receiver and an optimum exist for a medium swirling flow corresponding to a port angle of $\alpha=20^{\circ}$.

\subsection{Instantaneous swirl center locations}

In this section the instantaneous location of the swirl center is presented. The location is expressed as the distance from the instantaneous swirl center to the mean swirl center given by

$r_{\mathrm{sw}}=\sqrt{\left(x_{\mathrm{sw}}-\overline{x_{\mathrm{sw}}}\right)^{2}+\left(y_{\mathrm{sw}}-\overline{y_{\mathrm{sw}}}\right)^{2}}$

Table 3 Normalized static gauge pressures

\begin{tabular}{lllcl}
\hline$\alpha\left(^{\circ}\right)$ & $P_{1}^{*}$ & $P_{2}^{*}-P_{1}^{*}$ & $P_{3}^{*}-P_{2}^{*}$ & $P_{3}^{*}$ \\
\hline 0 & -2.88 & -49.3 & 5.3 & -46.9 \\
10 & -2.93 & -47.4 & 6.0 & -44.4 \\
20 & -3.16 & -47.2 & 8.1 & -42.3 \\
30 & -3.62 & -49.8 & 10.0 & -43.5 \\
\hline
\end{tabular}

The normalized pressures are given by $P_{i}^{*}=P_{i}\left(\frac{1}{2} \rho W_{b}^{2}\right)^{-1}$ where $\left(x_{\mathrm{sw}}, y_{\mathrm{sw}}\right)$ is the location of the instantaneous swirl center and $\left(\overline{x_{\mathrm{sw}}}, \overline{y_{\mathrm{sw}}}\right)$ is the mean swirl center location. It is noted that at the position $z / D=4.80$ in the $\alpha=10^{\circ}$ case, it is impossible to identify a swirl center in the majority of the snapshots since the in-plane velocities at this position are small and the velocity field disorganized.

Accurate location of the swirl centers is also impossible at positions $z / D=0.58$ and 1.00 for the $20^{\circ}$ and $30^{\circ}$ port angles. The swirl center in these cases is often significantly elongated and deformed, and in some cases multiple swirl centers are observed (see Fig. 5). Similar behavior of the vortex core is reported by Alekseenko et al. (1999) using visualization in a water-based swirl chamber. It has previously been shown that double or triple helical vortex structures in some cases exist in the vortex breakdown region in swirling flows (Sørensen et al. 2006; Ruith et al. 2003). Furthermore, preliminary results of proper orthogonal decomposition (POD) analysis (not shown) of the velocity fields confirm that multiple azimuthal modes exist at these positions. It is therefore expected that the observed vortex core deformations and occurrences of multiple vortex cores are the result of helical structures in the breakdown region. However, a full investigation of these phenomena is beyond the scope of this paper.

The instantaneous swirl centers are presented in Fig. 6 for the three port angles. For the $\alpha=10^{\circ}$ case, it is noted that the instantaneous swirl centers are located in a circular region around the mean swirl center. The circular region increases as a function of the downstream position, showing that the flow becomes increasingly unstable. As will be shown later in Sect. 3.6, no precession frequency is found at the downstream positions, indicating that the variation in the swirl center position for the $10^{\circ}$ case is random in nature. For the $20^{\circ}$ case the swirl centers are also observed to be located in a circular region around the mean swirl center for $z / D=[1.50,4.00]$. However, in this case, only a modest increase in size is observed for $z / D=1.50-3.50$,

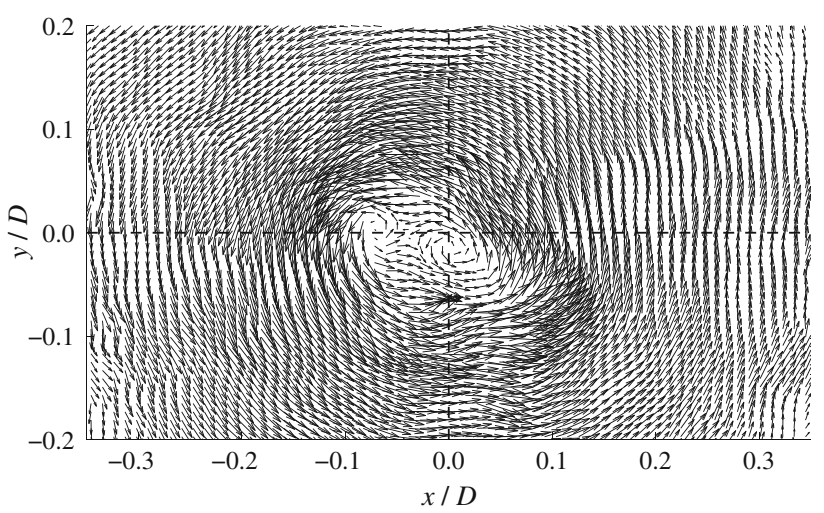

Fig. 5 Example of instantaneous in-plane vector field with two swirl centers. Data from $z / D=1.00$ with a port angle of $\alpha=20^{\circ}$ 


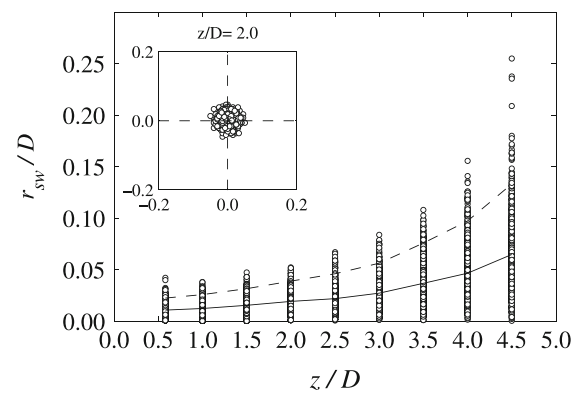

(a) $\alpha=10^{\circ}$

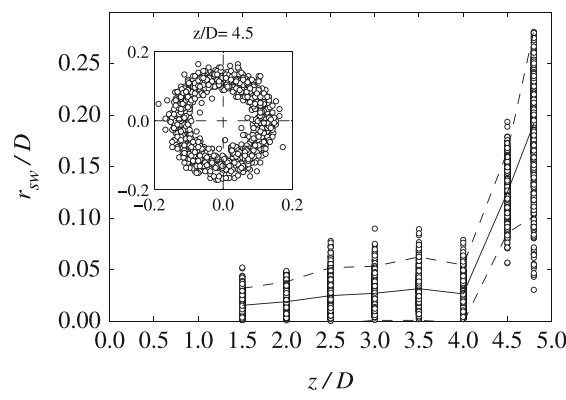

(b) $\alpha=20^{\circ}$

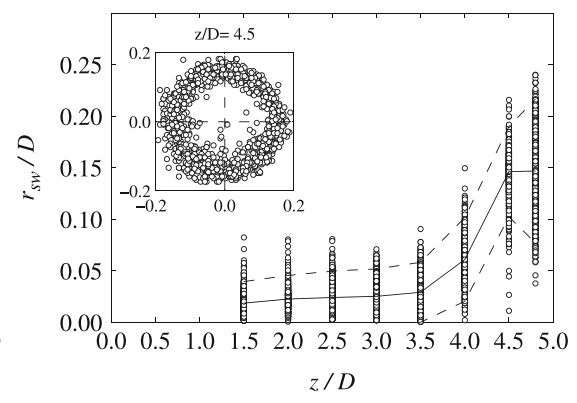

(c) $\alpha=30^{\circ}$

Fig. 6 Instantaneous swirl center position expressed as distance from mean swirl center position. Instantaneous swirl center location (circles), average distance from mean swirl center (-), and $95 \%$ confidence interval (- -)

and for $z / D=4.00$ the radius of the circular region is reduced compared to the $z / D=3.50$. For the $z / D=\{4.50$, $4.80\}$ positions, the swirl centers are located in an annular region with a mean radius of $r_{\mathrm{sw}} / D=0.12$ and $r_{\mathrm{sw}} / D=0.20$, respectively. This indicates that significant vortex precession occurs at these positions which is supported by LDA measurements. The large precession radius at $z / D=4.80$ further indicates that the vortex in the $\alpha=20^{\circ}$ case bends around the valve and continues out the exhaust pipe. The swirl center locations for the $30^{\circ}$ case are similar to the $20^{\circ}$ case for positions $z / D=[1.50,4.50]$, except for the fact that the swirl centers are located in an annular region already at $z / D=4.00$. As opposed to the $20^{\circ}$ case, it is observed that the mean precession radius decreases from $z / D=4.50$ to $z / D=4.80$, indicating that the vortex core does not bend around the outlet valve to the same degree as in the $20^{\circ}$ case.

\subsection{Non-swirling case $\left(\alpha=0^{\circ}\right)$}

Radial profiles of the mean radial and axial velocity are presented in Fig. 7 for the non-swirling case with a port angle $\alpha=0^{\circ}$. The corresponding rms-velocity profiles are included in Online Resource 1. Note that the profiles are mirrored in the cylinder axis in order to enhance the visual interpretation of the results. The axial profile at $z / D=0.58$ shows a nearly uniform velocity for $r / D=[0.00,0.35]$, with slightly lower velocities near the center. For $r / D>0.35$ the velocity shows a decrease as a function of $r$ which is the result of a separation at the wall just downstream the ports. The axial profiles become more uniform for the downstream positions up to $z / D=4.50$. At $z / D=4.80$ the axial profile is affected by the valve, resulting in low velocities near the center and the maximum velocity located at a radial position slightly larger than the exhaust valve radius. The radial velocities are in general much smaller than the axial velocities. At $z / D=0.58$ the radial velocities are negative for all radial positions except close to the center. This indicates that the flow has not completed the turn from flowing in the $x, y$-plane in the ports to flowing parallel to the $z$-axis in the cylinder. For positions of $z / D=1.00$ and $z / D=1.50$, the radial velocity is close to zero near the center, while small positive values are observed near the wall. This indicates that the flow reattaches to the wall downstream the port separation zone. For $z / D=4.50$ the radial velocities are now positive for all radial positions, indicating that the flow is starting to move outward in order to pass the valve. The tangential velocities are, as expected, small for all positions with a maximum absolute value smaller than $0.05 W_{b}$ and are therefore not shown.

\subsection{Swirling flow cases $\left(\alpha=10^{\circ}, 20^{\circ}, 30^{\circ}\right)$}

In this section the mean flow field is presented for the three swirling cases $\left(\alpha=10^{\circ}, 20^{\circ}, 30^{\circ}\right)$. Radial profiles of mean velocities $V_{r}, V_{\theta}, V_{z}$ and mean axial vorticity $\omega_{z}$ are presented in Figs. 8, 9, and 10. As for the non-swirling case, the profiles are mirrored around $r=0$ in order to enhance the visual interpretation. Note that the $V_{\theta}$ profiles are mirrored around both $r=0$ and the datum line. The corresponding rms-velocity profiles are available in Online Resource 1 . The profiles of axial vorticity are calculated from the profiles of tangential velocity as

$\omega_{z}(r)=\frac{1}{r} \frac{\mathrm{d}\left(\mathrm{rV}_{\theta}(\mathrm{r})\right)}{\mathrm{d} r}$

For each axial position two profiles are presented, one based on the mean swirl center and one based on the instantaneous swirl centers (cf. Sect. 2.6.1). Due to the problems in accurate location of the instantaneous swirl centers for positions $z / D=0.58$ and $z / D=1.00$ in the $\alpha=20^{\circ}$ and $\alpha=30^{\circ}$ cases, only profiles based on the mean swirl center are presented for these positions. Together with the profiles, iso-contours of the stream function $\psi$ are included in order to visualize the flow topology. The stream function is calculated by (Mattner et al. 2002) 
Fig. 7 Mean profiles of radial and axial velocity for a port angle of $\alpha=0^{\circ}$. The vertical dotted line corresponds to $V_{r} / W_{b}=0.25$ and $V_{z} / W_{b}=1.00$
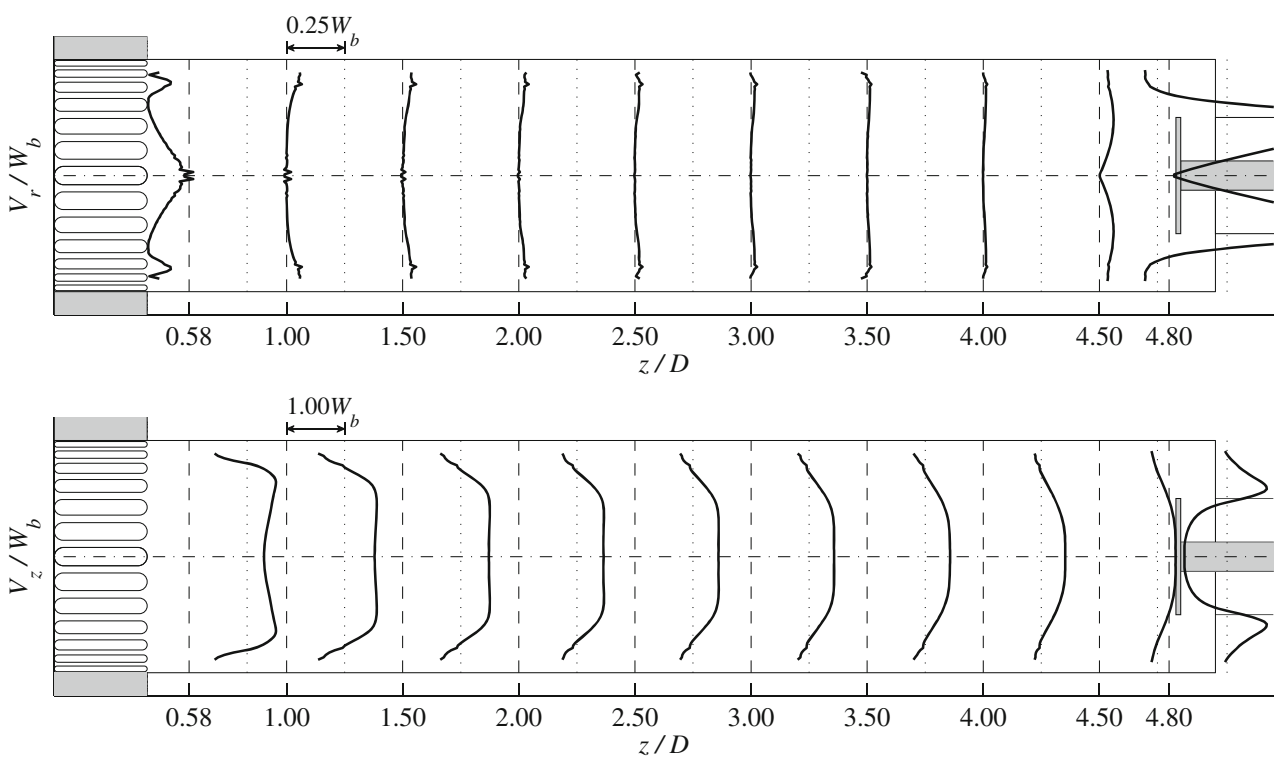

$\psi(r)=\int_{0}^{r} \hat{r} V_{z}(\hat{r}) \mathrm{d} \hat{r}$

using the axial profiles based on the mean swirl center. In the following a description of each case is presented.

\subsubsection{Port angle $\alpha=10^{\circ}$}

It is observed that a limited difference exists between profiles based on mean and instantaneous swirl centers. The differences between profiles are largest for axial positions in the last half of the cylinder. For the tangential velocity, the profiles based on instantaneous swirl centers show slightly larger values of the maximum tangential velocity $V_{\theta, \max }(z)$ and higher gradients close to the center, which also result in higher maximum values of $\omega_{z}$. For the axial velocities, the profiles based on instantaneous swirl centers show slightly lower centerline velocities. As both types of profiles show similar trends, a common discussion is presented. Studying the tangential profile (Fig. 8b) at $z / D=0.58$, it is observed that the profile corresponds to a concentrated vortex. The velocity increases close to linearly from zero at the center to $V_{\theta, \max }$ located close to the center. The radial position corresponding to $V_{\theta \text {,max }}$ is referred to as the vortex core radius. For larger radial positions the velocity decreases reaching approximately zero near the wall. For the downstream positions the vortex core radius increases, the maximum tangential velocity decreases, and constant velocities are observed at larger radial positions. This behavior corresponds to a vortex decaying as it moves downstream in the cylinder. The axial velocity (Fig. 8c) at the $z / D=0.58$ position is observed to be similar to the $0^{\circ}$ case except for a localized velocity deficit close to the center. The deficit increases from $z / D=0.58$ to 1.00 , showing both an increase in width and a lower centerline velocity. From $z / D=1.00$ and onward, the centerline velocity increases and the profiles become more uniform. The radial velocity profiles (Fig. 8a) are also similar to the $\alpha=0^{\circ}$ case. The main difference is that for $z / D=0.58$, zero or even small positive values are observed near the wall. The profiles of axial vorticity $\omega_{z}$ (Fig. 8d) show that the vorticity is confined to small region close to the center. Outside this region, the flow is irrotational as the values of $\omega_{z}$ are approximately zero. The maximum vorticity is observed at the center, and the vorticity decreases monotonically as a function of $r$. It is observed that the value of maximum vorticity decreases and the size of the central region increases as a function of downstream position. This behavior is again consistent with a decay of the vortex. The iso-contour plot of the stream function (Fig. 8e) shows that the flow is slightly divergent in the first part of the cylinder as it reattaches to the wall after the port recirculation zone. At axial positions near the valve, the streamlines close to the center start to bend outward such that the flow can go around the valve. It is noted that the streamlines do not show a central recirculation zone.

\subsubsection{Port angle $\alpha=20^{\circ}$}

The tangential profiles (Fig. 9b) in the first half of the cylinder are similar to the $\alpha=10^{\circ}$ case; however, both the vortex core radius and $V_{\theta, \max }$ are larger. At $z / D=2.00 \mathrm{a}$ significant decrease in the vortex core radius is observed. For the downstream positions, the profiles based on the instantaneous swirl centers show higher $V_{\theta \text {, max }}$ and smaller vortex core compared to profiles based on the mean swirl center. This effect is especially pronounced at $z / D=4.50$ 
Fig. 8 Radial profiles of mean velocities $\left(V_{r}, V_{\theta}, V_{z}\right)$, mean axial vorticity $\left(\omega_{z}\right)$, and isocontours of the stream function for a port angle of $\alpha=10^{\circ}$. The vertical dotted lines correspond to $V_{r} / W_{b}=0.25, V_{\theta} / W_{b}=$ $V_{z} / W_{b}=1.00$, and $\omega_{z} D / W_{b}=50$. (black curves) Profiles based on mean fields, (red curves) profiles based on mean fields using instantaneous swirl centers
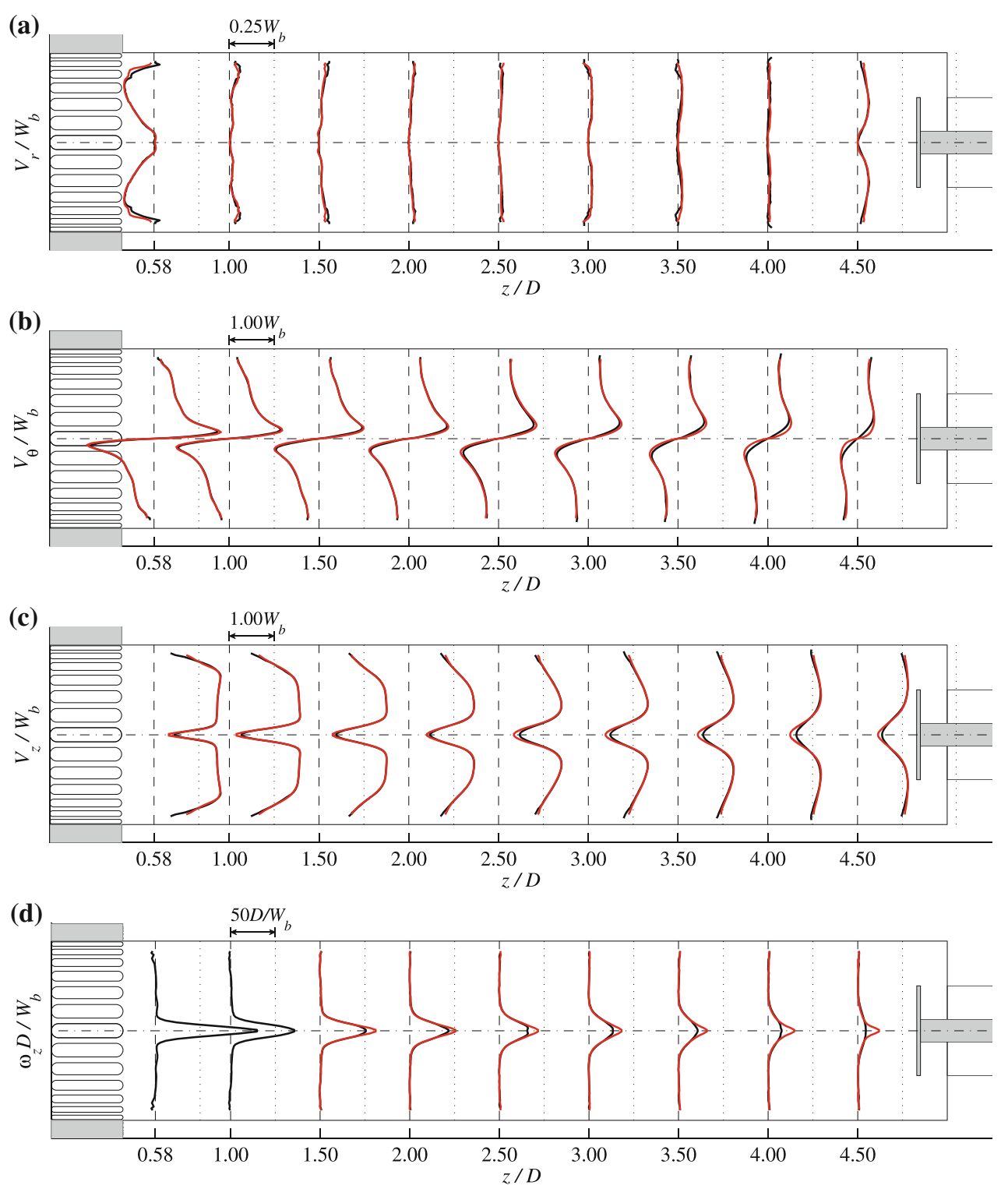

(e)

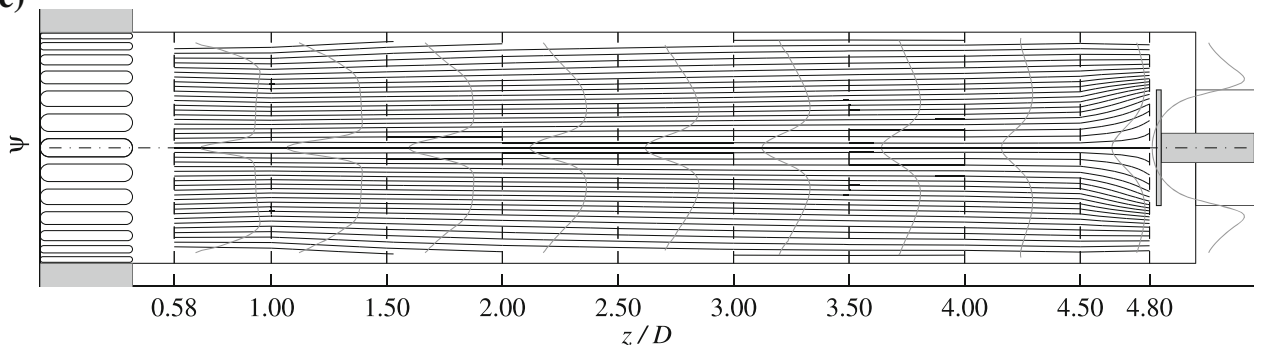

where significant vortex core precession occurs. The instantaneous profiles thus show that the vortex does not decay but remains strong and concentrated throughout the cylinder. If only profiles based on the mean swirl center were available, they could easily have been misinterpreted giving the impression that the vortex showed a rapid decay at $z / D=4.50$ due to interaction with the valve.
The axial velocity profiles (Fig. 9c) at the first three axial positions show a larger velocity deficit compared to the $10^{\circ}$ case. Furthermore, negative velocities are observed close to the center, showing that a vortex breakdown exists. For positions downstream the breakdown, a local maximum in the axial velocity profile is observed at the flow center $(r=0)$. This feature exists in 
Fig. 9 Radial profiles of mean velocities $\left(V_{r}, V_{\theta}, V_{z}\right)$, mean axial vorticity $\left(\omega_{z}\right)$, and isocontours of the stream function for a port angle of $\alpha=20^{\circ}$. The vertical dotted lines correspond to $V_{r} / W_{b}=0.25, V_{\theta} / W_{b}=$ $V_{z} / W_{b}=1.00$, and $\omega_{z} D / W_{b}=50$. (black curves) Profiles based on mean fields, (red curves) profiles based on mean fields using instantaneous swirl centers
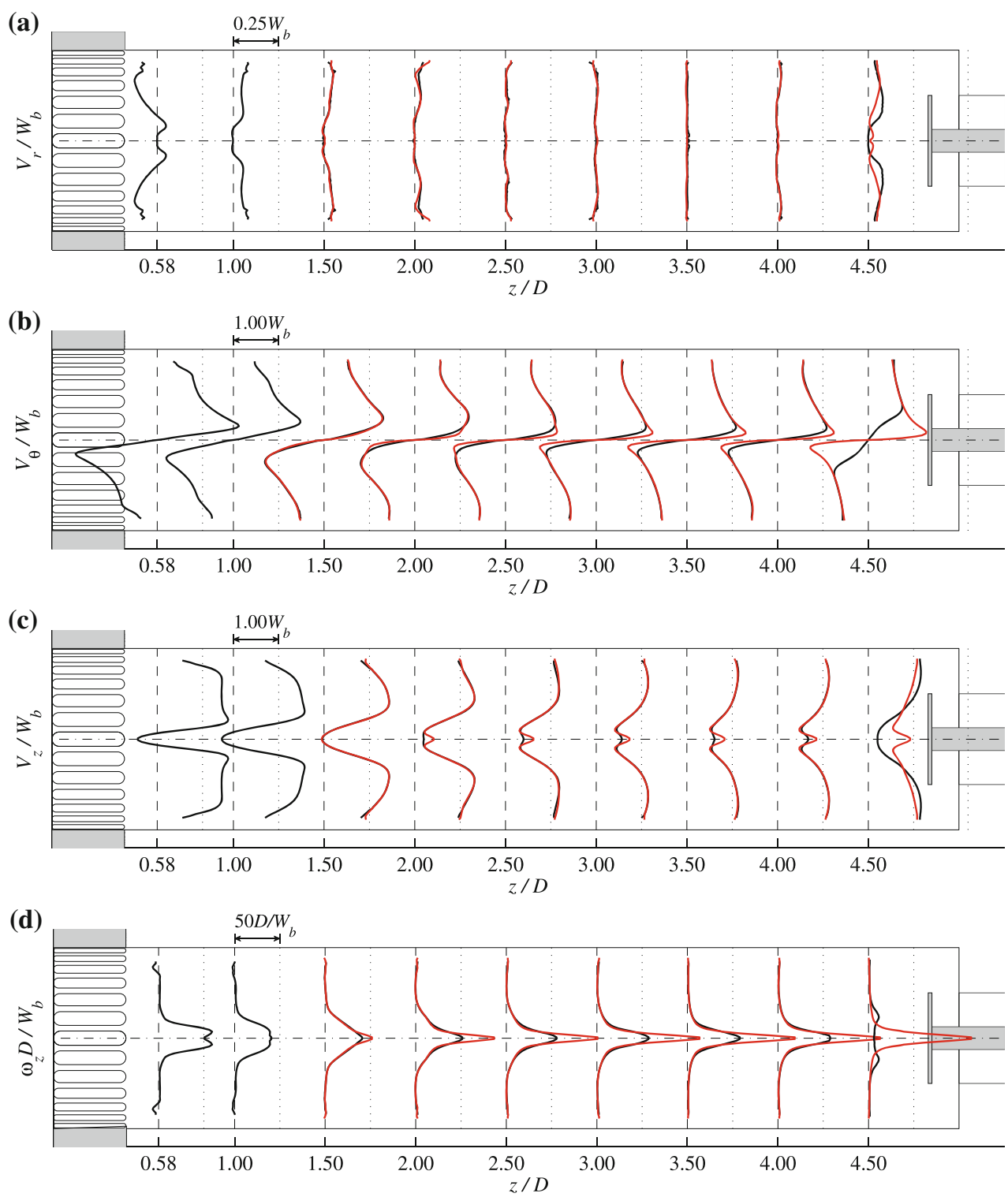

(e)

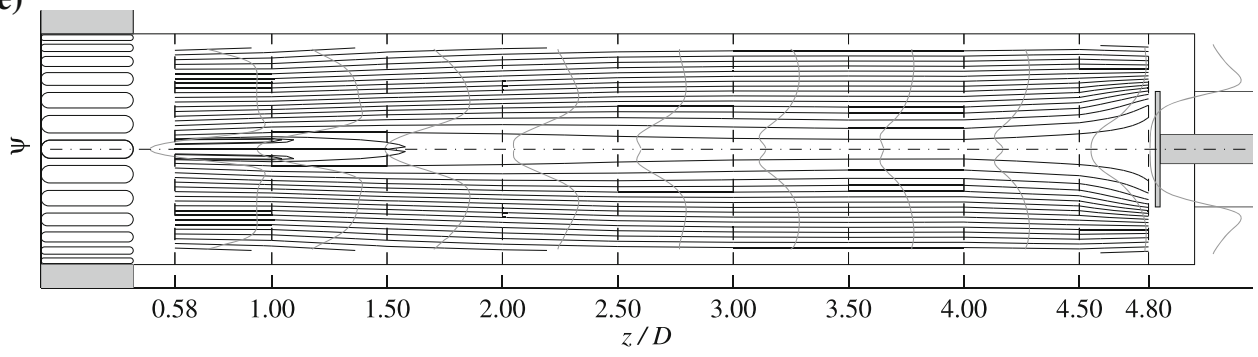

both types of profiles but is more pronounced in the profiles based on the instantaneous swirl centers. The peak in the axial velocity is expected to be the result of a suction effect created by a negative axial pressure gradient at the swirl center. The pressure gradient is caused by the acceleration of the fluid as it goes into the outlet contraction. Similar flow features have been reported by, for example, Escudier and Keller (1985), Escudier et al.
(2006), and Mattner et al. (2002); however, in these cases there were no obstacles in front of the outlet. It is therefore remarkable to observe the suction effect in the present geometry as the exhaust valve disk is blocking the direct access to the outlet and forcing the vortex to bend around the valve disc. Nonetheless, the basic mechanism is the same as in cases without an obstacle in front of the outlet. 
Fig. 10 Radial profiles of mean velocities $\left(V_{r}, V_{\theta}, V_{z}\right)$, mean axial vorticity $\left(\omega_{z}\right)$, and isocontours of the stream function for a port angle of $\alpha=30^{\circ}$. The vertical dotted lines correspond to $V_{r} / W_{b}=0.25, V_{\theta} / W_{b}=$ $V_{z} / W_{b}=1.00$, and $\omega_{z} D / W_{b}=50$. (black curves) Profiles based on mean fields, (red curves) profiles based on mean fields using instantaneous swirl centers
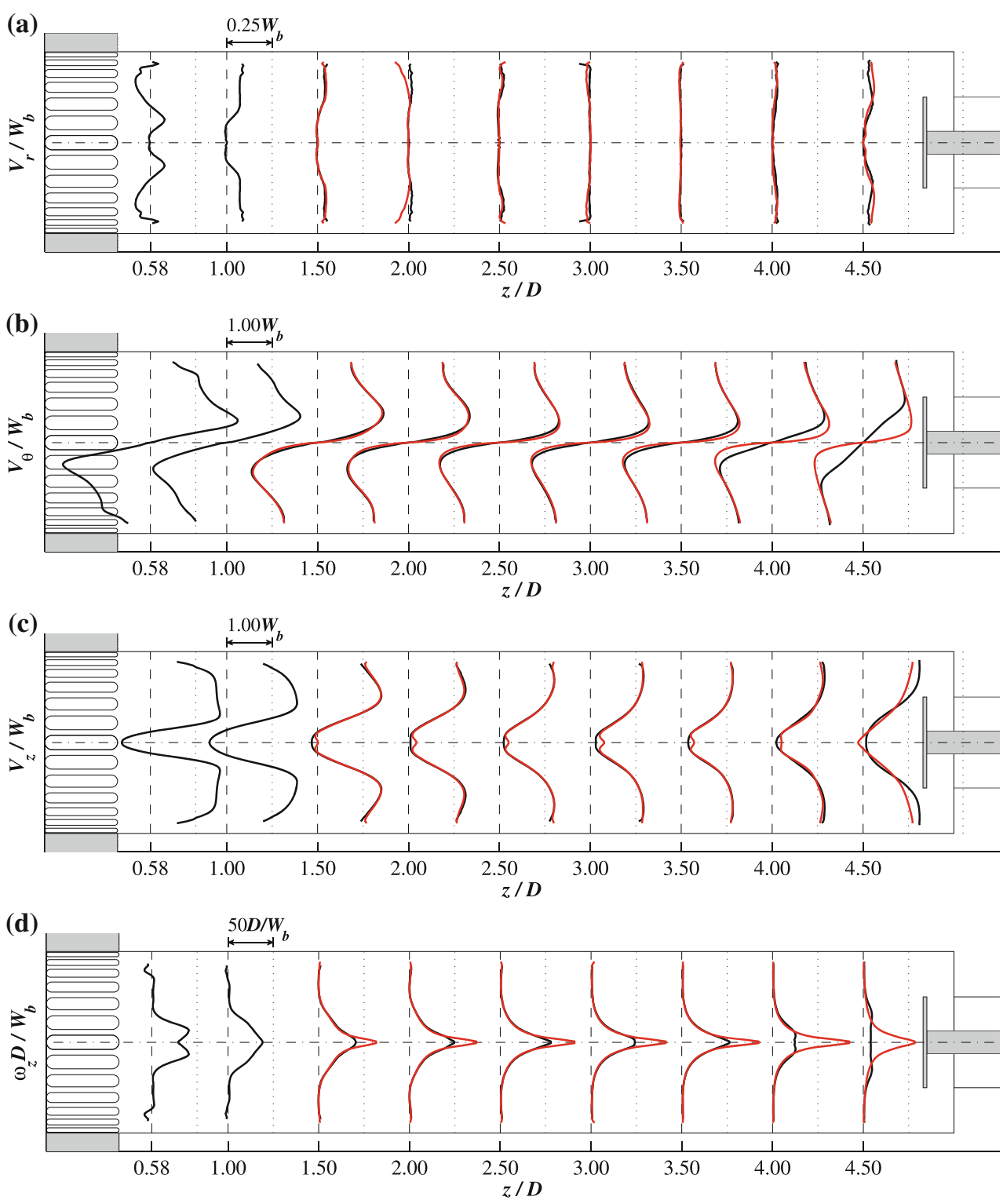

(e)

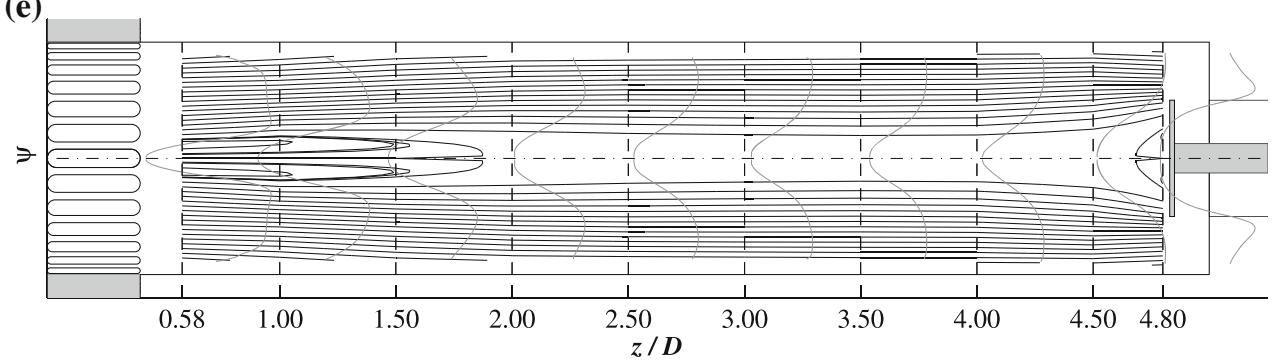

Studying the radial profiles (Fig. 9a), it is noted that the vortex breakdown region results in positive radial velocities near the center at the first axial position. Furthermore, it is noted that the positive velocities near the cylinder wall for $z / D=\{1.00,1.50\}$ have increased compared to the $10^{\circ}$ case, indicating that the recirculation zone downstream the ports has increased in size.
Similar to the $10^{\circ}$ case, the axial vorticity profile (Fig. 9d) at $z / D=0.58$ is characterized by a large peak around $r=0$. However, in the $20^{\circ}$ case a local deficit is observed at the very center; the deficit is expected to be the result of the large ratio of tangent circle diameter to port width. The deficit shows that the tangential velocity profile at $z / D=0.58$ does not correspond to a concentrated vortex 
but is a mix between a concentrated vortex and a wall-jet (Steenbergen and Voskamp 1998). For the downstream positions the deficit disappears and the profiles have the maximum value at the center. The width of the peak in the profiles decreases and the maximum value increases as a function of downstream position, indicating that the vortex gets more concentrated. From the iso-contours of the stream function $\psi$ (Fig. 9e), the size and shape of the vortex breakdown region are clearly observed. The recirculation extends to $r / D=0.06$ in the radial direction and $z / D=1.60$ in the axial direction.

The increase in swirl strength in the $20^{\circ}$ case compared to the $10^{\circ}$ case thus results in a vortex breakdown manifested as a central toroidal recirculation zone. Furthermore, the vortex does not decay after the breakdown but gets increasingly more concentrated, and a suction effect is observed at the vortex center. The fact that the vortex becomes concentrated and the presence of the suction effect are the results of an interaction with the outlet and thus show that the downstream conditions can affect the flow at a significant distance upstream. The ability of the downstream geometry to influence the flow field a significant distance upstream is reported and discussed by, for example, Escudier et al. (2006) and explained using the theory by Benjamin (1962). The theory states that the introduction of a sufficient amount of swirl causes a change in the flow state from a supercritical state to a subcritical state. The subcritical state is characterized by the ability of inertial waves to propagate upstream, which in practice means that the downstream conditions can influence the upstream flow field. The vortex breakdown is the transition from the supercritical to the subcritical state. The flow can then either return to the supercritical state after the breakdown or, if the swirl is sufficiently strong, remain subcritical a significant distance downstream.

In the context of the above-described theory, the flow in the $20^{\circ}$ case is expected to be a case where the flow transitions to the subcritical state near the ports and remains subcritical throughout the cylinder.

\subsubsection{Port angle $\alpha=30^{\circ}$}

In general, it is observed that both the profiles and the isocontours show trends similar to the $20^{\circ}$ case. For the tangential profiles (Fig. 10b), the increased port angle leads to larger tangential velocities and larger vortex core radius. This is especially clear for the profiles close to the ports. Furthermore, the degree to which the vortex becomes more concentrated after the breakdown is smaller compared to the $20^{\circ}$ case. The velocity deficit in the axial velocity profiles (Fig. 10c) has increased becoming both wider and with lower centerline velocities. It is noted that the local peak at the center of the axial velocity profile is less pronounced in the $30^{\circ}$ case. This corresponds well with the findings from Sect. 3.2, showing that the vortex does not bend around the exhaust valve to the same degree as in the $20^{\circ}$ case. For the $z / D=4.50$ position the peak in the axial velocity no longer exists, and negative axial velocities are observed in the flow center, indicating that a recirculation zone exists just upstream the valve. For the radial profiles (Fig. 10a) the only noticeable difference is that the positive velocities near the center at the first axial position have increased, indicating that the size of the breakdown region has increased. The profiles of axial vorticity (Fig. 10d) clearly show that the vortex in the $30^{\circ}$ case is less concentrated compared to the $20^{\circ}$ case as the maximum values are smaller and the region with significant positive vorticity has increased in size. The deficit observed in the peak at the first axial position has increased, supporting the claim that this is a direct effect of the size of the tangent circle. As expected from the profiles, iso-contours of the stream function (Fig. 10e) show that the vortex breakdown region has increased in both radial and axial direction. The breakdown region now extends to $r / D=0.10$ in the radial direction and $z / D=1.90$ in the axial direction.

\subsection{Angular momentum flux}

In this section the axial flux of angular momentum is estimated from the radial profiles of $V_{\theta}$ and $V_{z}$ and compared with the design swirl number $\Omega$. The axial flux of angular momentum is given by

$F_{\theta, z}=\int_{A_{c y l}} \rho V_{\theta} V_{z} r \mathrm{~d} A_{c y l}=\int_{0}^{D / 2} \rho V_{\theta}(r) V_{z}(r) r^{2} 2 \pi \mathrm{d} r$

To evaluate $F_{\theta, z}$ the velocity profiles must be extended to the wall, taking into account the boundary layer thickness $\delta$. The extension is performed by extrapolating the profiles to $r=\frac{D}{2}-\delta$, followed by a linear interpolation from $r=\frac{D}{2}-\delta$ to $r=\frac{D}{2}$ assuming that the velocity is zero at the wall. The boundary layer thickness $\delta$ is chosen such that integration of $V_{z}(r)$ over the cylinder cross section yields the bulk velocity obtained from the orifice flow meter, that is, $A_{c y l}^{-1} \int_{A_{c y l}} V_{z}(r) \mathrm{d} A=W_{b}$, leading to $\delta / D=3.5 \%$. The axial flux of angular momentum $F_{\theta, z}$ is presented as a function of the axial position in Fig. 11a. In the figure, $F_{\theta, z}$ is normalized using the axial flux of axial momentum corresponding to a plug flow $\left(F_{z, z}=\rho W_{b}^{2} A\right)$ and is therefore directly comparable to the design swirl number $\Omega$. It is observed that for $\alpha=\left\{10^{\circ}, 20^{\circ}, 30^{\circ}\right\}$ the flux of angular momentum shows a linear decrease as a function of axial position. The observed swirl decay is expected to be the result of viscous dissipation. In Fig. 11b the flux of angular momentum is plotted as a function of $\sin \alpha . F_{\theta, z}$ shows a close to linear 


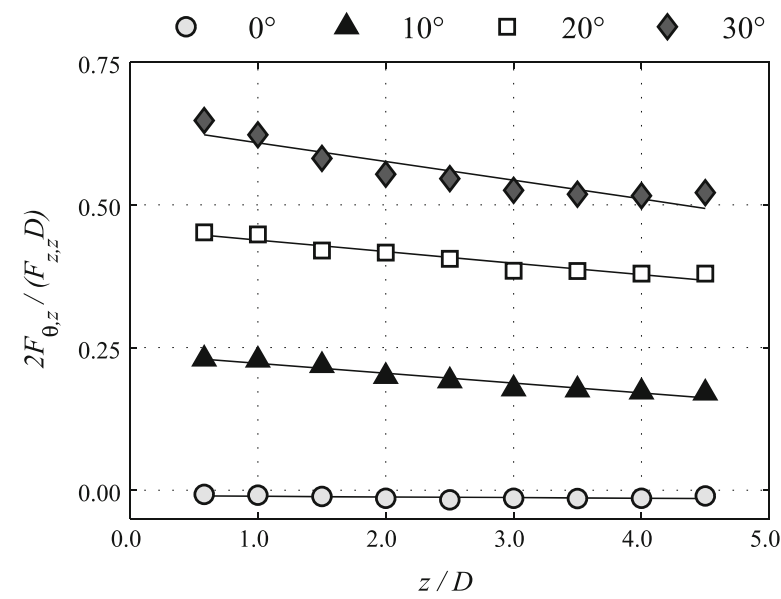

(a)

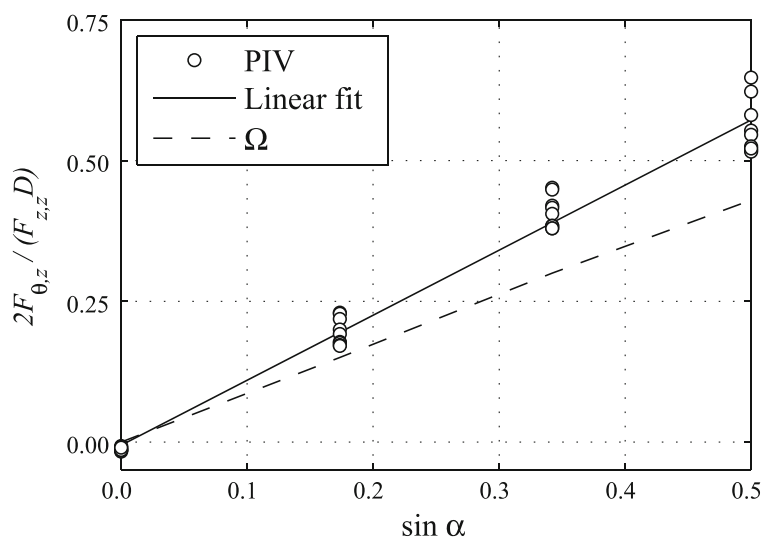

(b)

Fig. 11 Axial flux of angular momentum a normalized axial flux of angular momentum as a function of axial position for $\alpha=\left\{0^{\circ}, 10^{\circ}, 20^{\circ}, 30^{\circ}\right\}$, b normalized axial flux of angular momentum as a function of $\sin \alpha$, where $\alpha$ is the port angle

increase as a function of $\sin \alpha$ which is in agreement with the geometric analysis of the swirl number presented in Sect. 2.2. However, it is noted that the flux of angular momentum based on the PIV profiles is approximately $30 \%$ higher than estimated by the design swirl number. It is expected that the underestimation of $F_{\theta, z}$ based on the geometry is the result of a flow separation in the ports. The separated region in the ports results in a reduced effective flow area which leads to higher velocities at the port exit and hence higher radial flux of angular momentum.

\subsection{Power spectra}

A series of LDA measurements are carried out in order to study the precession of the vortex core. Measurements are performed at four horizontal positions $x / D=\{0.000,0.125,0.250,0.375\}$ for axial positions at $z / D=\{1.00,2.00,3.00,4.00\}$. For each measurement point a time series of the axial velocity component $v_{z}(t)$ is acquired. The length of the time series is $10 \mathrm{~min}$, and the mean acquisition rate is between 2.0 and $6.0 \mathrm{kHz}$ for all measured positions. The power spectrum $S$ is calculated using the method presented in Velte and George (2010), which takes into account the random arrival time of particles and the bias toward higher velocities. To reduce the noise of the spectra, block averaging is carried out using blocks of $2 \mathrm{~s}$ corresponding to 300 blocks.

In general, no clear peaks are observed in the case of the $0^{\circ}$ or $10^{\circ}$ ports, indicating that no significant vortex precession or other periodic phenomena occur in these cases. However, a small energy increase is observed for the $(x, z) / D=(0.0,1.0)$ point in the $10^{\circ}$ case (see Fig. 12a). The energy increase corresponds to a nondimensional frequency of $f D / W_{b} \approx 0.22$ and is expected to be a signature of a weak precession of the vortex on the piston surface. This is supported by the POD analysis of the PIV data (not included), showing that the dominant modes at $z / D=\{0.58,1.00\}$ correspond to in-plane displacements of the vortex core.

For the $\alpha=20^{\circ}$ and $30^{\circ}$ cases weak peaks are observed in the spectra for positions near the vortex breakdown region (not shown). The peaks correspond to frequencies in the range $f D / W_{b} \approx[2.0,10]$ and are expected to be related to the helical structures in the breakdown region discussed in Sect. 3.2. Well-defined high-energy peaks are observed in the spectra at $z / D=4.00$ for both the $\alpha=20^{\circ}$ and $30^{\circ}$ cases (see Fig. 12b, c, respectively). The swirl center locations presented in Sect. 3.2 showed that significant vortex core precession occurs at axial positions near the valve. The observed high-energy peaks are therefore expected to be the signature of the precessing vortex core (PVC). The PVC frequencies are $f D / W_{b}=0.5$ and $f D / W_{b}=0.48$ for the $\alpha=20^{\circ}$ and $30^{\circ}$ case, respectively. For the $\alpha=20^{\circ}$ case, the precession frequency is only observed for the $z / D=4.0$ plane. For the $\alpha=30^{\circ}$ case the peak corresponding to the PVC appears in the spectra for all four axial position; however, the energy of the peak decreases with upstream position. This indicates that the PVC affects a larger region of the in-cylinder flow for the $30^{\circ}$ case compared to the $20^{\circ}$ case. Alekseenko et al. (1999) find that the precession frequency increases linearly with the swirl number for swirl numbers smaller than 4.0. This does, however, not apply in the present case. The difference in observed behavior for the present case is expected to be caused by the different outlet geometry, that is, the valve. It is also noted that the spectra for the $30^{\circ}$ case show a peak at twice the PVC frequency. This is a result of the axial velocity field having the local peak in the swirl center surrounded by an annular region of low velocity, c.f, Fig. 13. As the vortex core rotates past the measurement volume, the low-velocity region is measured twice due to the annular shape resulting in the observed second peak. 


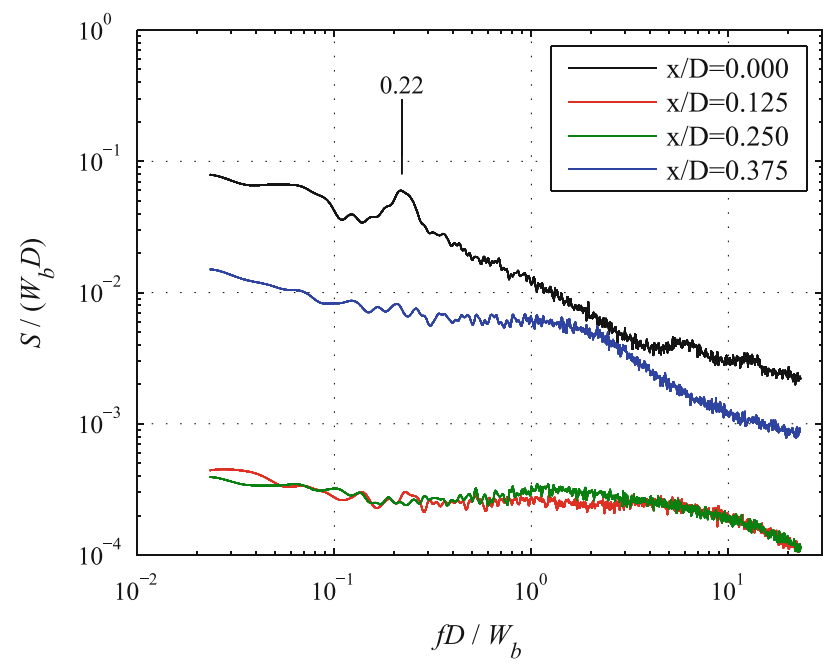

(a) $\alpha=10^{\circ}, z / D=1.0$

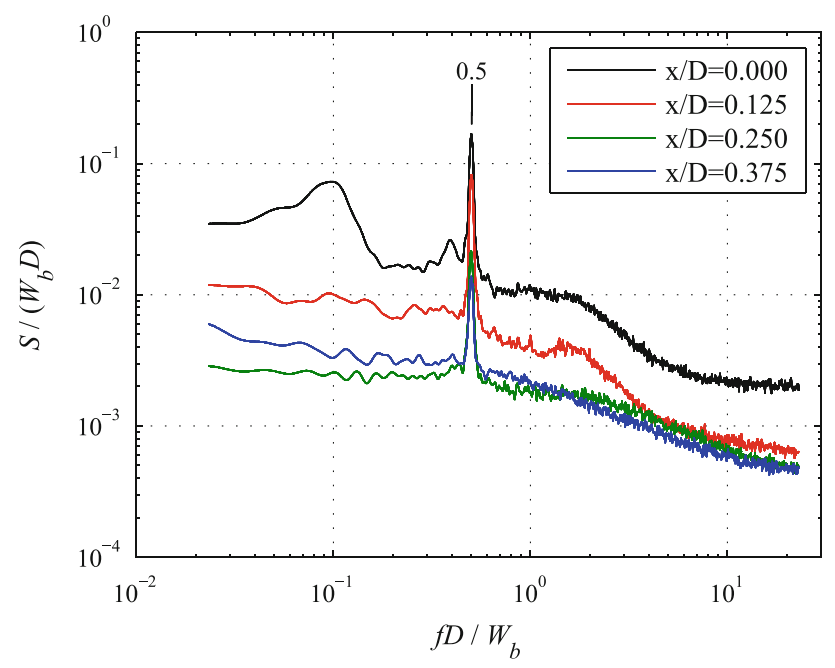

(b) $\alpha=20^{\circ}, z / D=4.0$

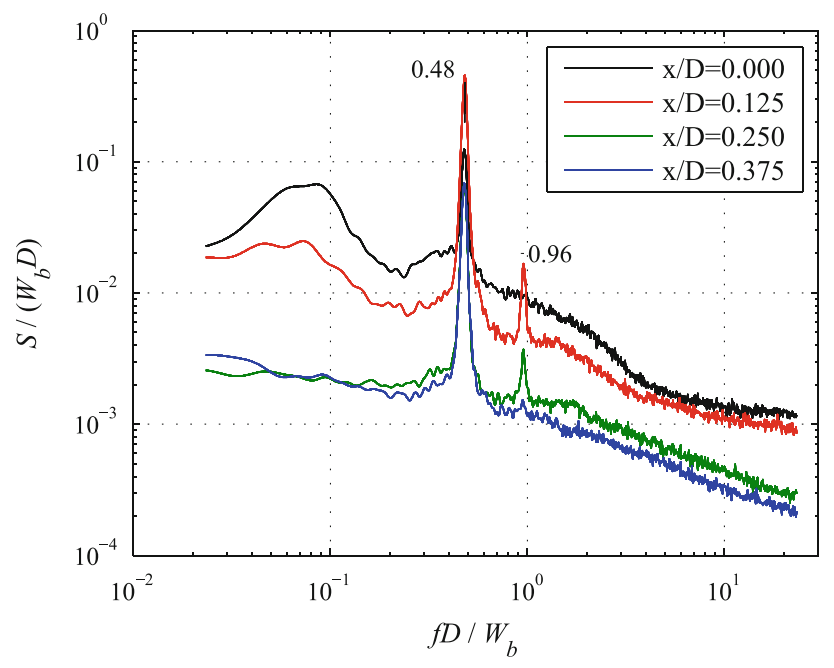

(c) $\alpha=30^{\circ}, z / D=4.0$

Fig. 12 Non-dimensional power spectra of $v_{z}(t)$

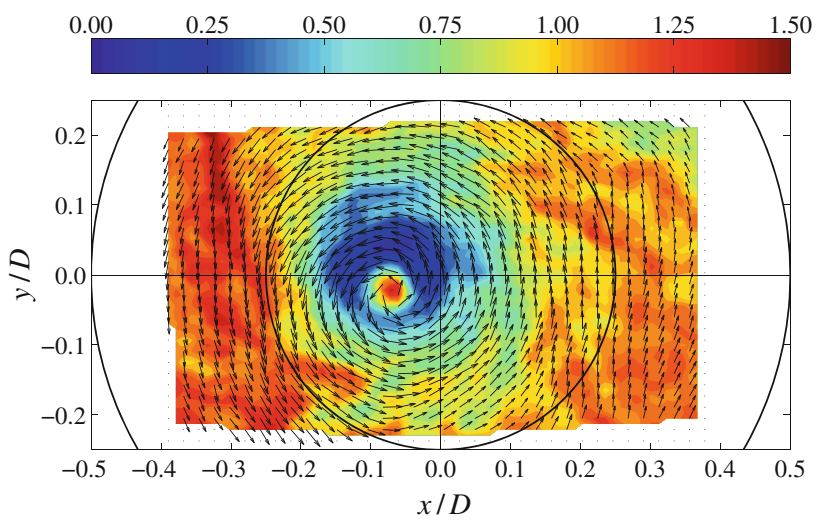

Fig. 13 Instantaneous velocity field for $\alpha=30^{\circ}$ at $z / D=4.00$. In-plane velocities $(u, v)$ are shown by vectors, and normalized axial velocity $w / W_{b}$ is shown by the contour plot. Every second vector is shown in each direction, and $W_{b}$ corresponds to a vector length of $0.025 D$ on the axis

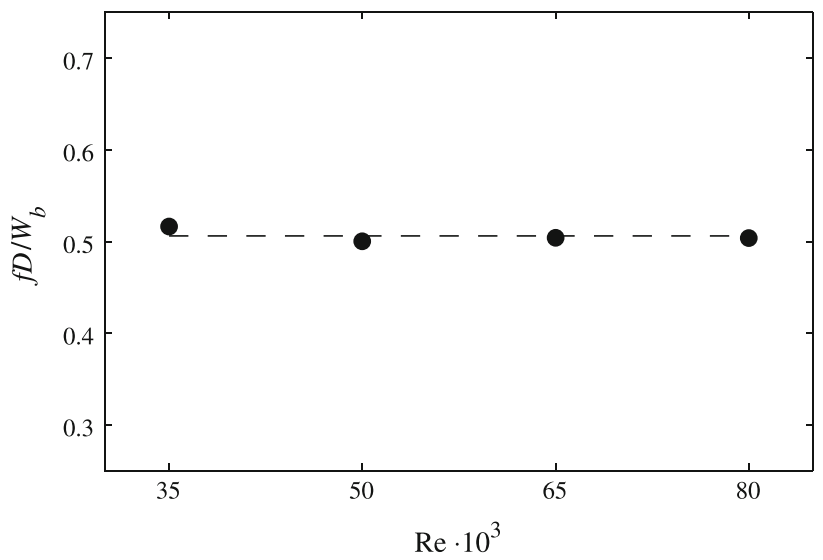

Fig. 14 PVC frequency as a function of Reynolds number for the $\alpha=20^{\circ}$ case

To investigate how the PVC frequency is affected by the bulk velocity, additional measurements are carried out for the $\alpha=20^{\circ}$ case. Measurements are carried out for the measurement point $(x, z) / D=(0.0,4.0)$ with bulk velocities corresponding to Reynolds numbers of $R e=$ $\{35,50,65,80\} \cdot 10^{3}$. The non-dimensional PVC frequency as a function of Reynolds number is presented in Fig. 14. The non-dimensional frequency is independent of the Reynolds number, and it can thus be concluded that the PVC frequency scales linearly with the bulk velocity. This is in agreement with Alekseenko et al. (1999) and references therein.

\section{Conclusion}

An experimental investigation of the turbulent swirling flow in a uniflow-scavenged two-stroke cylinder is carried 
out. Steady flow measurements are performed with stereoscopic particle image velocimetry and laser Doppler velocimetry. The measurements are carried out at a Reynolds number of $R e=D W_{b} / v=50,000$, where $D$ is the cylinder diameter, $W_{b}$ is the bulk velocity, and $v$ is the kinematic viscosity. In addition to standard profiles of mean velocity based on a fixed coordinate system, additional profiles are calculated based on the instantaneous flow topology using the instantaneous swirl centers as the origin for the coordinate system. The main findings of the work are as follows:

- The $10^{\circ}$ ports generate a highly concentrated vortex that decays as a function of downstream position. The decaying vortex flow is unstable with large random variations in the location of the instantaneous swirl centers. The introduction of swirl results in a local velocity deficit in the axial velocity profiles that does not exist in the non-swirling case.

- The increased swirl strength in the cases with port angles $\alpha=20^{\circ}$ and $30^{\circ}$ results in the formation of a central toroidal recirculation zone. The recirculation is elongated in the axial direction compared to the radial and results in reversed flow near the center. The recirculation zone is interpreted as the manifestation of a vortex breakdown region.

- For the $\alpha=20^{\circ}$ and $30^{\circ}$ cases, the vortex becomes highly concentrated downstream the vortex breakdown and shows significant vortex core precession at positions close to the valve. This is a result of an interaction with the downstream conditions and indicates that the in-cylinder flow is in a subcritical state for angles of $\alpha=20^{\circ}$ and above.

- The concentrated and precessing vortices in the $\alpha=20^{\circ}$ and $30^{\circ}$ cases result in a suction effect along the vortex core, which causes a local peak to appear in the center of the axial velocity deficit.

- The Strouhal number $S t=f D / W_{b}$, where $f$ is the precession frequency, is found to be approximately 0.5 for both the $20^{\circ}$ and $30^{\circ}$ cases, and thus independent of the swirl number. Furthermore, the Strouhal number is found to be independent of the Reynolds number.

- Profiles based on the instantaneous flow topology are shown to be more representative of the in-cylinder flow compared to standard profiles in cases with high gradients and variations in the instantaneous swirl center location.

Acknowledgments Financial funding from the Danish Agency for Science Technology and Innovation (Grants No. 09-070608, MAN Diesel \& Turbo SE) is greatly acknowledged, as well as fruitful discussions with Jens Nørkær Sørensen, Valery Okulov, and Poul Scheel Larsen.

\section{References}

Albrecht HE, Damaschke N, Borys M, Tropea C (2003) Laser Doppler and phase Doppler measurement techniques. Springer, Berlin

Alekseenko SV, Koubin PA, Okulov VL, Shtork SI (1999) Helical vortices in swirl flow. J Fluid Mech 382:195-243

Benjamin TB (1962) Theory of the vortex breakdown phenomenon. J Fluid Mech 14(4):593-629

Craft T, Iacovides H, Launder B, Zacharos A (2008) Some swirlingflow challenges for turbulent CFD. Flow, Turbul Combust 80:419-434

Dedeoglu N (1990) Improvement of mixture formation in a uniflowscavenged two-stroke engine. SAE Tech Paper Ser Paper No. 901536

Escudier MP, Keller JJ (1985) Recirculation in swirling flow: a manifestation of vortex breakdown. AIAA J 23(1):111-116

Escudier MP, Bornstein J, Maxworthy T (1982) The dynamics of confined vortices. Proc R Soc Lond A 382:335-350

Escudier MP, Nickson AK, Poole RJ (2006) Influence of outlet geometry on strongly swirling turbulent flow through a circular tube. Phys Fluids 18:125103

Gupta AK, Lilley DG, Syred N (1984) Swirl flows. Abacus Press, Tunbridge Wells

Haider S, Schnipper T, Obeidat A, Meyer KE, Okulov VL, Mayer S, Walther JH (2012) PIV study of the effect of piston position on the in-cylinder swirling flow during the scavenging process in large two-stroke marine diesel engines. J Mar Sci Tech accepted

Holman JP, Moore GD (1961) An experimental study of vortex chamber flow. J Basic Eng 83(4):632-636

Huang RF, Tsai FC (2001) Observations of swirling flows behind circular disks. AIAA J 39(6):1006-1112

Ingvorsen KM, Meyer KE, Schnipper T, Walther JH, Mayer S (2012) Swirling flow in model of large two-stroke diesel engine. In: 16th international symposium on applications of laser techniques to fluid mechanics, Lisbon, Portugal

Litke B (1999) The influence of inlet angles in inlet ports on the scavenging process in two-stroke uniflow-scavenged engine. Mar Tech III 45:247-252

Mattner TW, Joubert PN, Chong MS (2002) Vortical flow. Part 1. Flow through a constant-diameter pipe. J Fluid Mech 463: 259-291

Nakagawa H, Kato S, Tateishi M, Adachi T, Tsujimura H, Nakashima M (1990) Airflow in the cylinder of a 2-stoke cycle uniform scavenging diesel engine during compression stroke. Jpn Soc Mech Eng 33(3):591-598

Nishimoto K, Kamimoto T (1984) A study on the influence of inlet angle and Reynolds number on the flow-pattern of uniflow scavenging air. SAE Tech Paper Ser 93(4):4788-4797, Paper No. 841056

Ohigashi S, Kashiwada Y, Achiwa J (1960) Scavenging the 2-stroke engine-effect of inlet port-angle on scavenging process of a through scavenging system. Jpn Soc Mech Eng 3(9):130-136

Percival WH (1955) Method of scavenging analysis for 2-stroke-cycle diesel cylinders. SAE Trans 63:737-751

Raffel M, Willert CE, Wereley ST, Kompenhans J (2007) Particle image velocimetry. A practical guide, 2nd edn. Springer, Berlin

Ruith MR, Chen P, Meiburg E, Maxworthy T (2003) Threedimensional vortex breakdown in swirling jets and wakes: direct numerical simulation. J Fluid Mech 486:331-378

Sanborn D, Dedeoglu N (1988) Investigation on scavenging of twostroke engines. SAE Tech Paper Ser Paper No. 881264

Sarpkaya T (1971) On stationary and travelling vortex breakdowns. J Fluid Mech 45(3):545-559 
Schweitzer PH (1949) Scavenging of two-stroke cycle diesel engines. Macmillan Publishing Company, London

Sher E, Hossain I, Zhang Q, Winterbone DE (1991) Calculation and measurements in the cylinder of a two-stroke uniflow-scavenged engine under steady flow conditions. Exp Therm Fluid Sci 4: $418-431$

Sørensen JN, Naumov I, Mikkelsen R (2006) Experimental investigation of three-dimensional flow instabilities in a rotating liddriven cavity. Exp Fluids 41:425-440

Steenbergen W, Voskamp J (1998) The rate of decay of swirl in turbulent pipe flow. Flow Meas Instrum 9(2):67-78
Sung NW, Patterson DJ (1982) Air motion in a two stroke engine cylinder-the effects of exhaust geometry. SAE Trans pp 2534-2544, Paper No. 820751

Velte CM, George WK (2010) Efficient estimation of burst-mode LDA power spectra. In: 15th international symposium on applications of laser techniques to fluid mechanics, Lisbon, Portugal, Paper No. 1652

Wakuri Y, Ono S, Kido H, Takasaki K (1981) An experimental study on the exhaust smoke of a diesel engine with variable angle swirler. Bull Jpn Soc Mech Eng 24(193):1198-1205, Paper No. 193-14 